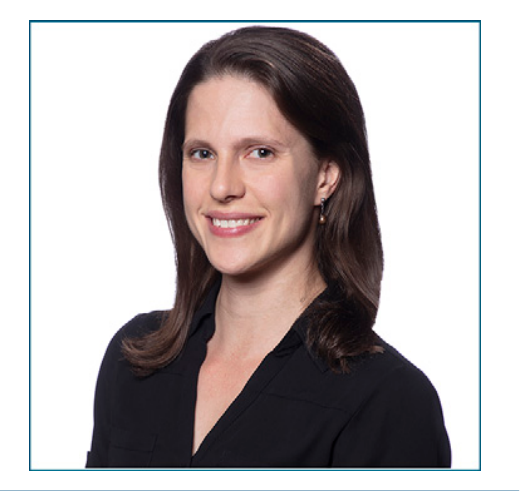

LIANNA J. MARKS, MD

Lianna J. Marks, MD, is a Clinical Assistant Professor of Pediatrics at the Stanford University School of Medicine in the Division of Pediatric Hematology, Oncology, Stem Cell Transplantation \& Regenerative Medicine. Her research interests include early phase clinical trials for pediatric patients with relapsed or refractory lymphoma and incorporating circulating tumor DNA analysis into clinical trials in pediatric lymphoma with the hope of improving risk stratification and response assessment. doi: 10.6004/jnccn.2021.7041

The ideas and viewpoints expressed in this commentary are those of the author and do not necessarily represent any policy, position, or program of NCCN.

\section{Improving Risk Stratification to Guide Treatment Decisions for Children, Adolescents, and Young Adults With Hodgkin Lymphoma}

\author{
Lianna J. Marks, MD
}

H odgkin lymphoma $(\mathrm{HL})$ is a highly curable disease with multiple evidencebased treatment options for children and adolescents. With the expectation of long-term survival of patients with $\mathrm{HL}$ comes the responsibility to use treatment regimens that reduce the risk of late effects, such as cardiovascular disease, infertility, pulmonary complications, and second malignant neoplasm (SMN). Recognizing the unique needs of this patient population, clinical trials developed for pediatric and adolescent patients embrace risk-adapted, response-based protocols focused on minimizing long-term toxicity while preserving high cure rates. The preferred primary treatment regimens recommended in the new NCCN Clinical Practice Guidelines in Oncology (NCCN Guidelines) for Pediatric Hodgkin Lymphoma, including the Children's Oncology Group (COG) studies AHOD0031 and AHOD1331 and the European Pediatric and Adolescent Hodgkin Lymphoma Network (EuroNet-HD) study EuroNet-PHL-C1, build on previous studies developed with these goals in mind. For adolescents and young adults (AYAs) ages 15 to 39 years, who make up the majority of patients with $\mathrm{HL}$, an important initial decision is whether to treat on pediatric or adult protocols. In general, the recommended approaches consist of several cycles (2-6) of multiagent chemotherapy with or without involved-site radiation therapy (ISRT), depending on initial risk group and interim PET response. Although enrollment on a phase III clinical trial is encouraged if available, choosing the best treatment approach for an individual patient can be a complex choice given the different treatment options and desire to balance individual risk factors and treatment toxicities.

Initial staging of patients with $\mathrm{HL}$ is the foundation for developing a riskadapted treatment plan. Both pediatric and adult groups rely on the Ann Arbor classification system for staging, but treatment approaches, risk stratification, and response criteria have evolved in parallel due to differences in clinical practice between disciplines. Following consensus efforts by adult lymphoma cooperative groups, the international Staging Evaluation and Response Criteria Harmonization (SEARCH) for Childhood, Adolescent and Young Adult Hodgkin Lymphoma (CAYAHL) group was formed to standardize the staging process and allow for improved comparisons of pediatric cooperative group trials. ${ }^{1}$ However, comparing outcomes between trials is further complicated by the differing risk stratification and group allocation approaches used not only by adult and pediatric cooperative group trials but also between pediatric HL groups and between generations of trials within the same group. The criteria for unfavorable clinical presentations used to determine risk stratification vary among protocols, but typically include stage, the presence of "B" symptoms, bulky lymphadenopathy, and extranodal extension to contiguous structures ( $E$ lesions). Of note, adult cooperative groups and the EuroNet-HD pediatric

See page 733 for related article. 
group incorporate erythrocyte sedimentation rate as a variable in risk group assignment. Risk group definitions frequently evolve from outcomes of previous related studies. For example, patients with stage IIB HL with bulk disease treated as intermediate risk on COG trial AHOD0031 had suboptimal outcomes compared with patient with stage IIB HL without bulk disease, and therefore on the subsequent high-risk study, AHOD1331, patients with stage IIB HL with bulk were considered high-risk (ClinicalTrials.gov identifier: NCT02166463). Although different risk group criteria make sense in the context of specific protocols, they lead to substantial patient heterogeneity across HL studies, making direct comparison of outcomes between groups a challenge.

For AYA patients who reasonably could be treated on pediatric or adult protocols, there can be significant variation in risk stratification. Based on current pediatric guidelines, an 18-year-old patient with stage IIIA HL is considered intermediate risk whether or not there is bulky disease, although the presence of E-lesions increases them to high risk according to EuroNet-PHL-C1. In contrast, if treated according to adult guidelines, any patient with stage III HL is considered to have advanced disease. These differences in risk stratification impact treatment duration and intensity. The optimal treatment of AYA patients remains a subject of debate. Studies evaluating outcomes for AYA patients treated on pediatric versus adult trials are limited. A recent analysis of patients with $\mathrm{HL}$ aged 17 to 21 with stage III/IV disease treated on ECOG Intergroup trial E2496 had significantly worse 5-year failure-free survival (FFS) and overall survival (OS) than patients in the same age group treated on COG AHOD0031 when controlled for stage, B symptoms, and bulk disease (5-year FFS, 68\% vs $81 \%$; OS, $89 \%$ vs $97 \% ; P<.0001)^{2}$

Although several retrospective trials have demonstrated similar outcomes between AYAs treated on adult protocols, their follow-up periods do not adequately capture the long-term treatment-related morbidity and mortality that is of particular concern in this patient population. ${ }^{3}$ Patients treated on COG AHOD0031 had a 10 -year cumulative incidence of SMN of $1.3 \%(95 \% \mathrm{Cl}$, $0.6 \%-2.0 \%$, with higher cumulative incidence of SMNs among patients who received radiation therapy (RT; $P=.037$ ), highlighting the potential benefit of protocols that omit RT while maintaining good disease control. ${ }^{4}$ Other factors, including risk of chest radiation in women, fertility concerns, treatment schedule, and underlying medical conditions, all influence treatment decisions for AYAs and must be considered when determining their treatment course and treatment setting.

Optimizing risk stratification at diagnosis guides therapy selection and is an initial step toward ensuring that an individual patient receives the appropriate amount of chemotherapy, neither being overtreated and risking excessive long-term toxicity, nor being undertreated and risking relapse, which would then require more toxic salvage therapy. Prognostic factors can assist in further refining treatment choice. The NCCN Guidelines for adult $\mathrm{HL}$ use the International Prognostic Score (IPS), defined by the number of adverse prognostic factors present at diagnosis, to help determine clinical management for patients with advanced-stage disease (stage III-IV). ${ }^{5}$ However, IPS predictors, such as age $\geq 45$ years, do not apply to the pediatric and adolescent population, and therefore the Childhood Hodgkin International Prognostic Score (CHIPS) was developed as a predictive model for eventfree survival in pediatric and adolescent $\mathrm{HL}$ and is being validated in a recently completed COG trial (NCTN 02166463). ${ }^{6}$

A better understanding of $\mathrm{HL}$ biology and validation of prognostic biomarkers across age groups could contribute to risk stratification and response assessment. A range of biomarkers investigating mutations in malignant cells, tumor microenvironment, and host immune response-related polymorphisms have been studied in adults with $\mathrm{HL}$; however, less is known about biomarkers in the pediatric and AYA populations. Although the knowledge gained from adult studies is relevant to pediatrics, any prognostic biomarkers identified need to be validated in pediatrics. Gene expression profiling in adults with advanced-stage $\mathrm{HL}$ identified a 23-gene signature predictive of survival, but preliminary evidence suggests this profile was not predictive in pediatric patients, possibly due to differences in biology or therapy. ${ }^{7}$ Circulating tumor DNA detected through next-generation sequencing is also emerging as a tool for identifying genomic mutations and improving risk stratification and response assessment in HL. Prospective studies evaluating potential biomarkers across the age spectrum are essential for ensuring that their use in risk stratification is applicable to all patients.

There is movement toward greater collaboration on clinical trials for diseases such as HL that span adult and pediatric age groups, including an intergroup phase III trial treating patients aged $\geq 12$ years with advanced-stage HL (ClinicalTrials.gov identifier: NCT03907488). This effort may increase access to novel therapies for pediatric patients, increase trial enrollment, and facilitate the study of biomarkers in different populations. However, it is critical that the motivation to decrease long-term toxicities in young patients with $\mathrm{HL}$ that informed prior pediatric protocols is not lost in translation. Current adult trials also use risk-adapted designs, refined RT strategies, and targeted therapies with the hope of reducing long-term toxicities. Nevertheless, the most frequently used adult chemotherapy backbone of ABVD (adriamycin/bleomycin/vinblastine/dacarbazine), included in the new NCCN Guidelines for Pediatric HL as an "other recommended" therapy, has much higher cumulative anthracycline and 
bleomycin doses compared with pediatric protocols including ABVE-PC (doxorubicin/bleomycin/vincristine/etoposide/prednisone/cyclophosphamide) and OEPA/ COPDac (vincristine/etoposide/prednisone/doxorubicin/ cyclophosphamide/dacarbazine) which were developed specifically to reduce cumulative doses and associated toxicities. Adult trials aimed at deescalating therapy, such as the Response-Adapted Therapy in Advanced Hodgkin Lymphoma (RATHL) trial, which omits bleomycin from the last 4 cycles for patients with a negative interim PET, still expose patients to high levels of anthracyclines. ${ }^{8}$ Both pediatric and adult trials have worked to identify patients with adequate responses to chemotherapy in whom RT can be safely omitted, but for patients requiring RT, the ISRT dose typically used in adults of 30 to $36 \mathrm{~Gy}$ is greater than the 21 Gy recommended in children. As more adolescents become eligible to enroll on trials with adult patients, considering the potential benefits of targeted therapies along with the risks of higher exposures to cytotoxic chemotherapy and RT will be important in determining optimal therapy.

New studies building upon data from long-term follow-up studies of patients treated for $\mathrm{HL}$ are improving understanding of cumulative burden of cardiovascular morbidity and other chronic health conditions, and may help guide up-front therapy decisions and protocol development. ${ }^{9}$ A recent report from the Childhood Cancer
Survivor Study demonstrated that risk-adapted therapy has successfully reduced the risk of serious late effects in patients with pediatric HL. ${ }^{10}$ Looking forward, the Hodgkin Lymphoma International Study for Individual Care (HoLISTIC) Consortium is developing dynamic decision models using individual patient data from recent large prospective trials in children and adults. This simulation modeling helps inform treatment decisions for patients with $\mathrm{HL}$, including AYAs, by considering short-term disease outcomes with estimates of absolute risks of late effects and impacts on health-related quality of life. ${ }^{11}$ The HoLISTIC Consortium data are also being used to create a modern prognostication index for patients with advanced-stage $\mathrm{HL}$ that may be applicable across ages. ${ }^{12}$ Ultimately, incorporating prognostic biomarkers and dynamic decision models may improve the precision of risk stratification and allow for better therapeutic tailoring of the intensity and duration of treatment based on individual relapse risk and long-term toxicities.

Disclosures: Dr. Marks has disclosed receiving no financial considerations from any person or organization to support the preparation, analysis, results, or discussion of this article.

Correspondence: Lianna J. Marks, MD, Stanford University School of Medicine, 1000 Welch Road, Suite 300, Stanford, CA 94304.

Email: marks|@stanford.edu

\section{References}

1. Flerlage JE, Kelly KM, Beishuizen A, et al. Staging Evaluation and Response Criteria Harmonization (SEARCH) for Childhood, Adolescent and Young Adult Hodgkin Lymphoma (CAYAHL): methodology statement. Pediatr Blood Cancer 2017;64:e26421.

2. Henderson TO, Parsons SK, Wroblewski KE, et al. Outcomes in adolescents and young adults with Hodgkin lymphoma treated on US cooperative group protocols: an adult intergroup (E2496) and Children's Oncology Group (COG AHOD0031) comparative analysis. Cancer 2018;124:136-144.

3. Foltz LM, Song KW, Connors JM. Hodgkin's lymphoma in adolescents. J Clin Oncol 2006;24:2520-2526.

4. Giulino-Roth L, Pei Q, Buxton A, et al. Subsequent malignant neoplasms among children with Hodgkin lymphoma: a report from the Children's Oncology Group. Blood 2021;137:1449-1456.

5. Hasenclever D, Diehl V. A prognostic score for advanced Hodgkin's disease. International Prognostic Factors Project on Advanced Hodgkin's Disease. N Engl J Med 1998;339:1506-1514.

6. Schwartz CL, Chen L, McCarten K, et al. Childhood Hodgkin International Prognostic Score (CHIPS) predicts event-free survival in Hodgkin lymphoma: a report from the Children's Oncology Group. Pediatr Blood Cancer 2017;64:e26278.
7. Mottok A, Johnston RL, Chun Chan F, et al. Prediction of primary treatment outcome using gene expression profiling of pre-treatment biopsies obtained from childhood and adolescent Hodgkin lymphoma patients. Blood 2015;126:175.

8. Johnson P, Federico M, Kirkwood A, et al. Adapted treatment guided by interim PET-CT scan in advanced Hodgkin's lymphoma. N Engl J Med 2016;374:2419-2429.

9. Bhakta N, Liu Q, Yeo F, et al. Cumulative burden of cardiovascular morbidity in paediatric, adolescent, and young adult survivors of Hodgkin's lymphoma: an analysis from the St Jude Lifetime Cohort Study. Lancet Oncol 2016;17:1325-1334.

10. Oeffinger KC, Stratton KL, Hudson MM, et al. Impact of risk-adapted therapy for pediatric Hodgkin lymphoma on risk of long-term morbidity: a report from the Childhood Cancer Survivor Study [published online February 25, 2021]. J Clin Oncol, doi: 10.1200/JCO.20.01186

11. Parsons SK, Kelly MJ, Cohen JT, et al. Early-stage Hodgkin lymphoma in the modern era: simulation modelling to delineate long-term patient outcomes. Br J Haematol 2018;182:212-221.

12. Rodday AM, Parsons SK, Scharman C, et al. Prognostication for advanced stage Hodgkin lymphoma $(\mathrm{HL})$ in the modern era: a project from the Hodgkin Lymphoma International Study for Individual Care (HoLISTIC) Consortium. Blood 2020;136(Suppl 1):16-18. 